\title{
Three Dimensional Simulation of Multiphase Immiscible Displacements Using a Combined Grid and Particle Approach
}

\author{
* Homolka D. T., * Archer J. S., ** Graham J. M. R., * Daltaban T. S. \\ Imperial College ; University of London \\ * Dept. Mineral Resources Engineering \\ ** Dept. Aeronautical Engineering
}

\begin{abstract}
Copyright 1995, Steering Committee of the European IOR - Symposium.
This paper was presented at the 8th. European IOR - Symposium in Vienne, Austria, May 16 - 17, 1995

This paper was selected for presentation by the Steering Committes, following review of information contained in an abstract submitted by the author(s). The paper, as presented has not been reviewed by the Steering Committee.
\end{abstract}

\section{ABSTRACT}

The aim of this work is to model complex petroleum reservoirs in terms of relevant but arbitrarily shaped heterogeneities. This removes the constraint of describing heterogeneities as constant property regions confined to cartesian grid blocks.

This paper describes the use of a particle method in tracking characteristics such as saturation fronts. Work, in two-dimensions, has been completed to allow the heterogeneity to be described in an arbitrary geometry, using boundary integral methods. The problem investigated is water displacing oil in a porous medium such as an oil reservoir.

The numerical method described for the solution of a three-dimensional, two-phase immiscible displacement (waterflood model), involves utilising a large number (i.e. several hundreds of thousands) of moving points to carry particular 'properties' of interest, such as water saturation. The property represented may then be transformed from the particles onto the fixed mesh where the equations of motion can be solved. Solving the transport equation on the mesh then allows the particles to be moved to their new positions.

Contrasting with previous, two-dimensional work, where particles have been used to represent passive concentration gradients, particles are used here to characterise saturation in three dimensions. The changing saturation on the mesh causes the pressure field, and hence fluid velocity to vary which causes certain problems which must be addressed. When considering the motion of discontinuities, biased interpolation methods, and some particle controls, are used to maintain accuracy. Sensitivity of the solution to differing methods of particle control was investigated and a selection of methods were evaluated. This combined grid/particle technique has successfully been used to reproduce, numerically, two dimensional displacement profiles, as well as analytic solutions of the Buckley-Leverett equation in one-dimension. Good agreement between numerical and analytical results has been found.

\section{Introduction}

Boundary integral methods have been used to model single-phase fluid flow in complex heterogeneous porous geometries [Homolka, 1995, and, Wong, 1991]. Motion of fronts describing the interface between two fluids of differing properties, was described by using vortex panels to represent the fluid interface.

In simulations of hydrocarbon reservoirs the fluids in place are very rarely, if ever, at $100 \%$ saturation of the pore space, thus at a particular point in the reservoir the fluid is considered to be a mixture of the fluid phase in place, and the displacing fluid phase. In this case it is more difficult to specify exact boundaries between regions containing fluids of different compositions.

The use of particle methods to model general convection-diffusion problems encountered in the study of fluid dynamics have previously been discussed quite extensively by Graham [1988], Farmer \& Norman [1986a], Raviart [1986], and, Christiansen [1973]. Also, the application of particle methods to the modelling of many body non-linear plasma problems has been investigated by Birdsall \& Fuss [1969]. 
The use of moving point methods similar to the particle methods used in fluid dynamics models have been described by Pietlicki \& Archer [1987] as a means of controlling numerical diffusion in reservoir simulation. Indeed, Graham [1988] mentions that one of the important advantages of particle methods is the reduction in numerical diffusion due to the Lagrangian formulation of the methods.

Farmer [1986b] also investigated the use of particle methods for solving convection diffusion equations. The case considered was that of moving points being used to represent a concentration gradient of a fluid within a second transporting fluid. The velocity of the particles was taken to be the characteristic convection velocity, and diffusion was calculated by allowing the value of concentration represented by a particle to change over time.

\section{Multi-Phase Fluid Flow}

When considering hydrocarbon recovery processes, single phase flow models are of limited interest. A model that is considered useful is a two phase system where water is the displacing fluid phase and oil is the fluid phase in place. The fluid composition at any point in the reservoir is described in terms of the saturations of each of the phases. In general, in a two phase case, prior to any fluid displacement the reservoir will be full of fluid consisting of both water at the 'connate' or 'irreducible' water saturation $\left(S_{w_{i r}}\right)$ and oil at a saturation of $\left(1-S_{w_{i r}}\right)$.

Aziz \& Settari [1979] describe the derivation of equations representing multi-phase flow in three dimensions resulting in equations of conservation,

$$
\begin{aligned}
& \nabla \cdot\left[\lambda_{o}\left(\nabla p_{o}-\gamma_{o} \nabla z\right)\right]=\frac{\partial}{\partial t}\left[\frac{\phi S_{o}}{B_{o}}\right]+q_{o} \\
& \nabla \cdot\left[\lambda_{w}\left(\nabla p_{w}-\gamma_{w} \nabla z\right)\right]=\frac{\partial}{\partial t}\left[\frac{\phi S_{w}}{B_{w}}\right]+q_{w} \\
& \nabla \cdot\left[R_{s} \lambda_{o}\left(\nabla p_{o}-\gamma_{o} \nabla z\right)+\lambda_{g}\left(\nabla p_{g}-\gamma_{g} \nabla z\right)\right] \\
& =\frac{\partial}{\partial t}\left[\phi\left(\frac{R_{s} S_{o}}{B_{o}}+\frac{S_{g}}{B_{g}}\right)\right]+R_{s} q_{o}+q_{f g}
\end{aligned}
$$

where, $\lambda_{i}$, the transmissibilities, are defined as,

$$
\lambda_{i}=\frac{k_{H}}{\mu_{t} B_{i}} k
$$

where, $k$ is the absolute permeability of the porous medium,

$k_{r l}$ is the relative permeability of the material to phase $l$,

$\mu_{l}$ is the viscosity of phase $l$, and

$B_{l}$ is the formation volume factor of phase $l$, i.e. the ratio between a volume of fluid at reservoir conditions of temperature and pressure and its volume at a standard condition.

In addition, the follow conditions also apply,

$$
\begin{aligned}
& S_{o}+S_{w}+S_{g}=1 \\
& P_{c o w}=p_{o}-p_{w}=f\left(S_{w}, S_{g}\right)
\end{aligned}
$$

$$
P_{c o g}=p_{g}-p_{o}=f\left(S_{w}, S_{g}\right)
$$

The capillary pressure functions are usually empinical relationships derived from laboratory experiments.

The conservation equations for incompressible flow, assuming negligible rock compressibility may be written in the form,

$$
\begin{aligned}
& -\nabla \cdot\left[\tilde{\lambda} \frac{d P_{c}}{d S_{w}} \nabla S_{w}\right]-\left[{\underset{\sim}{u}}_{T} \frac{d f_{w}}{d S_{w}}+\Delta \gamma \nabla z \frac{d \tilde{\lambda}}{d S_{w}}\right] \cdot \nabla S_{w} \\
& =\phi \frac{\partial}{\partial t} S_{w}+q_{w}-f_{w} q_{T}
\end{aligned}
$$

Equation (8) is generally parabolic, because $\frac{d P_{c}}{d S_{w}}<0$, and becomes hyperbolic if $P_{c}=0$, in which case,

$$
-\left[{\underset{\sim}{u}}_{T} \frac{d f_{w}}{d S_{w}}+\Delta \gamma \nabla z \frac{d \bar{\lambda}}{d S_{w}}\right] \cdot \nabla S_{w}=\phi \frac{\partial}{\partial t} S_{w}+q_{w}-f_{w} q_{T}
$$

which is a hyperbolic equation, because $\frac{d f_{w}}{d S_{w}}>0$.

If the fluids have equal density, (or $\nabla z=0$ ), then the fractional flow equation simplifies to,

$$
\underline{\sim}_{n}=f_{n} \underline{\sim}_{T} \text { and } \underline{u}_{w}=f_{w} \underline{u}_{T}
$$

and equation (9) may then be written as,

$$
-\sim_{T} \frac{d f_{w}}{d S_{w}} \cdot \nabla S_{w}=\phi \frac{\partial}{\partial t} S_{w}+q_{w}-f_{w} q_{T}
$$

For the wetting phase, equation (8) may also be written as,

$$
\nabla \cdot\left[f_{w} \cdot{\underset{\sim}{u}}_{T}\right]-\nabla \cdot\left[\tilde{\lambda}\left(\nabla P_{c}\right)\right]=\phi \frac{\partial}{\partial t} S_{w}-q_{w}
$$

The first term on the left hand side is due to viscous forces, the convection term, and the second is due to capillary forces, the diffusion term.

\section{The Particle Method}

The particle method, also known as the cloud-in-cell method uses many thousands of particles in order to characterize the saturation variation of a two component fluid. The development of the method will be discussed in two dimensions, and the changes required to use the method in three dimensions will be indicated. Each of the particles in the flow domain is assigned a value of saturation, and, its motion is tracked through the domain. The motion of the particles is governed by the pressure field in the domain under consideration. The pressure field is subject to variation due to the distribution of the saturation, which is characterized by the particles. This method of representing and calculating changes in the saturation can be described to be an IMPES scheme.

Using the Biot-Savart integral, [Graham, 1988], it is possible to calculate the interaction between each pair of particles. However this is only feasible for relatively small numbers of particles, $\left(N_{\text {par }}\right)$, as the Biot-Savart method requires of the order of $\mathrm{O}\left(N_{\text {parr }}^{2}\right)$ operations per time step. When a larger number of particles is used, in 
order to improve the representation of the saturation distribution, an alternative method of solution must be employed.

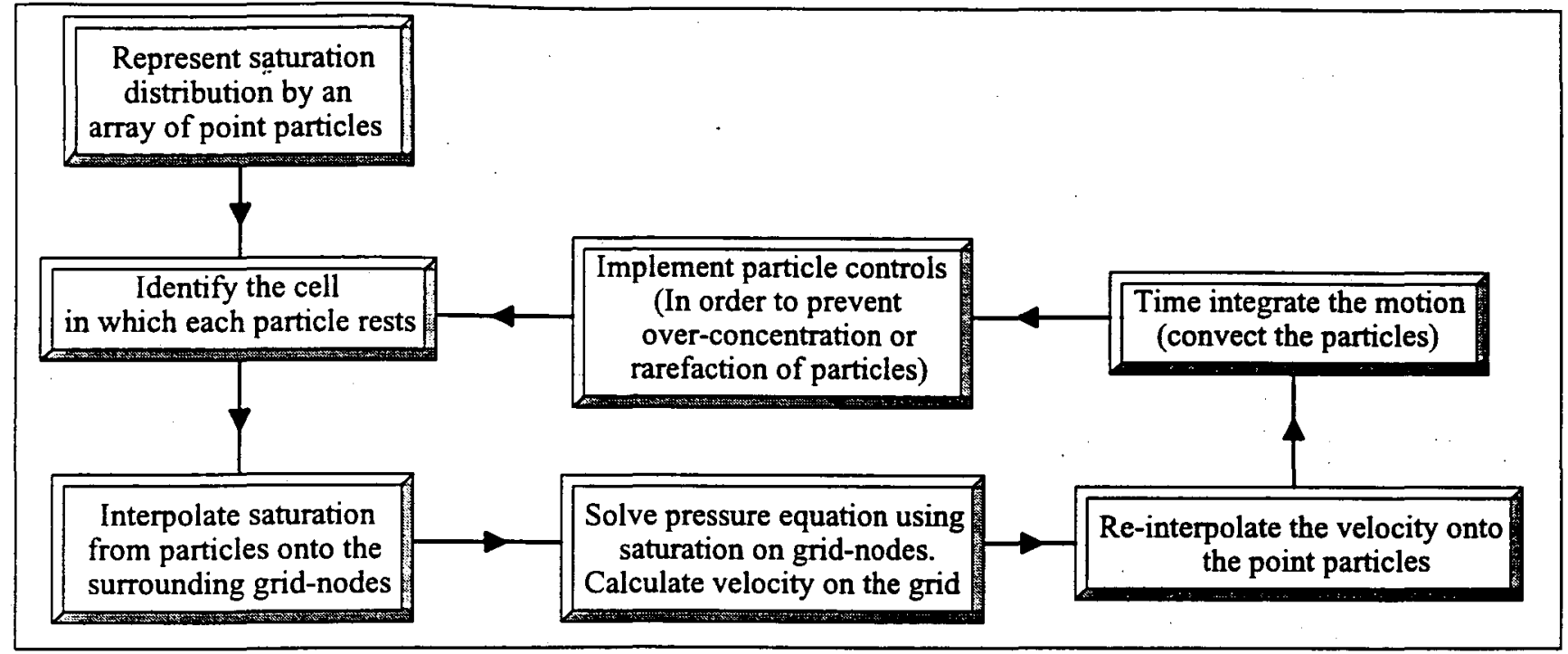

Figure 1. Evolution of saturation distribution.

One way to solve for large numbers of particles is to superimpose a grid onto the flow field and carry out some of the calculations on the grid. All of the quantities defined on the grid are assigned at the grid nodes. A gridblock may be thought to surround each node, making this scheme similar in terms of representation of properties to a block-centred finite difference system.

The value of saturation can be calculated at the grid nodes and the pressure equation can be solved on the grid. Once the pressure is known on the grid, it is possible to determine the value of the velocity at each grid node, and, using a weighting function, at each particle. Thus each particle may be convected to its new location, see Figure 1.

On a mesh of size $N_{x}$ by $N_{y}$, the number of operations required at each time step is of the order of $\mathrm{O}\left(N_{x}^{2} N_{y}^{2}\right)$. So provided $\left(N_{x} N_{y}\right)<<\left(N_{p a r}\right)$, the mesh scheme is more computationally efficient than the direct calculation scheme.

Although the mesh scheme has the advantage that for large numbers of particles it is very computationally efficient, it does introduce an element of numerical diffusion. The magnitude of the numerical diffusion is related to both the size of the time step, as well as the grid spacing.

This method of solution has been used to describe the evolution of vortex sheets by Birdsall \& Fuss [1969], and, by Christiansen [1973] in order to determine the motion of clouds of charged particles.

\subsection{Interpolation From Particles To Nodes}

In order to determine the appropriate saturation distribution on the grid, the values of saturation must be averaged onto the grid nodes from the particles. In order to do this, an opposite area weighting scheme is employed, see Figure 2.
The contribution of each particle to the value of saturation at a particular node point is calculated as follows. From Figure 2, the cell in which the particle rests is divided into four segments, each of area $A_{i} i=1$, $\ldots$,4. The cells are all of size $A_{\text {cell }}$, thus,

$$
A_{\text {cell }}=\sum_{i=1}^{4} A_{i}
$$

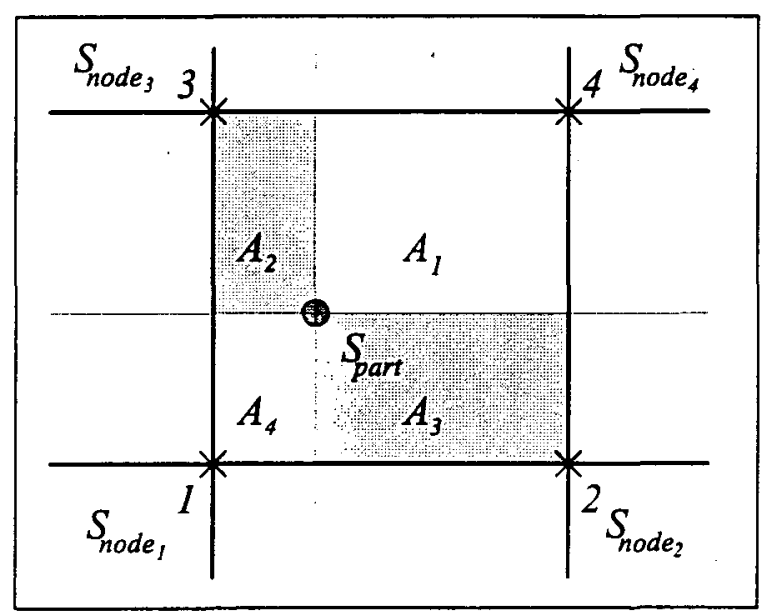

Figure 2. The opposite area weighting scheme employed to interpolate saturation from particles to grid nodes.

Each particle, of saturation $S_{\text {part }}$, contributes to the value of saturation at each of the grid nodes in the following proportions,

$$
S_{\text {node }_{i}}=S_{\text {part }} \frac{A_{i}}{A_{\text {cell }}}
$$

This contribution of each particle to the value of saturation at each grid node is also weighted in proportion to the total number of particles in the immediate area of the grid node.

This interpolation scheme has previously been used to model fluid flow using point vortices by Basuki [1983], 
and, Felix [1987]. The scheme is known to conserve vorticity, which is the property carried by the point vortices. Thus it is reasonable to assume that saturation is also conserved using this method of interpolation, which in turn means that mass is conserved.

Once the saturation is known at the grid nodes it is possible to solve the pressure equation (12) on the grid. Equation (12) may also be written as,

$$
\begin{aligned}
& -\nabla\left[f_{w} \lambda\left(S_{w}\right) \nabla p\right]-\nabla \cdot\left[\lambda\left(S_{w}\right) \nabla p_{c}\right] \\
& =\phi \frac{\partial}{\partial t} S_{w}-q_{w}
\end{aligned}
$$

which is equivalent to,

$$
\begin{aligned}
& -\nabla\left[f_{w} \lambda\left(S_{w}\right) \nabla p\right]-\nabla \cdot\left[\lambda\left(S_{w}\right) \nabla p_{c}\right] \\
& =-\phi \lambda\left(S_{w}\right) \nabla p \nabla S_{w}-q_{w}
\end{aligned}
$$

The pressure equation governs the movement of the particles. In order to calculate the convection velocity of each particle the total velocity at each grid node is first calculated from the pressure,

$$
v=-\lambda\left(S_{w}\right) \nabla p
$$

where, $\lambda\left(S_{w}\right)$ is the total mobility of the fluid at the point in space being considered. The equivalent finite difference equation is,

$$
v_{i j}=\left(\frac{\lambda\left(s_{w_{i j}}\right)\left(P_{i-1-j}-P_{i+1 j}\right)}{2 \Delta x}, \frac{\lambda\left(s_{w_{i j}}\right)\left(P_{i j-1}-P_{i j+1}\right)}{2 \Delta y}\right)
$$

The value of the total velocity vector at any point, $\mathbf{x}_{i j}$, within a particular grid cell is calculated using bilinear weighting, similar to the procedure adopted to interpolate saturation from the particles to grid nodes,

$$
v\left(\mathbf{x}_{i j}\right)=\sum_{i=1}^{4} v_{i} \frac{A_{i}}{A_{c e l l}}
$$

where, $v_{i}$ is the velocity at each of the vertices of the cell in which the particle rests.

Thus a particle of saturation $S_{\text {part }}$ will be convected with a velocity,

$$
v_{p}=\lambda\left(S_{\text {part }}\right)\left[\sum_{i=1}^{4} v_{i} \frac{A_{i}}{A_{c e l l}}\right]
$$

Once the particles have been moved to their new positions, it is possible to re-calculate the saturation at the grid nodes, ind the pressure solution on the grid. Oil and water production rates from the reservoir model are calculated at this point. Also any particle control that must be applied in order to assure the accuracy of the solution must be implemented.

The model initially implemented is the simple Buckley-Leverett two phase displacement. Capillary effects are not taken into account at this stage. A comparison is made of a Buckley-Leverett displacement calculated by the particle method to the solution obtained by Buckley \& Leverett [1942]. The effect of including the capillary pressure on the calculation is also investigated. Two dimensional displacements were also considered, and the results were compared to experimental work of Beadie \& Dawe [1982].

\subsection{Solving The Pressure Equation}

The pressure equation can be written as a Poisson equation (20). The right hand side, however, contains unknown terms that cause difficulty. This problem can be treated by using a fully implicit formulation of the equation, or by using previous time step information as an approximation to the true values. The Poisson formulation makes it possible to implement a fast solver based on the method of Fourier transforms [Cooley \& Tukey, 1965], as has been used in the field of aeronautical engineering by, for example, Basuki [1983] and Felix [1987]. The use of FFT to solve the pressure equation was investigated, [Homolka, 1995], but the solution was found to diverge for large discontinuities, and the method was replaced by a proven, stable, iterative solver based on Line Successive Over Relaxation, supplied by Daltaban [1994]. An iterative solver was chosen as direct methods of solution are field size limited.

The pressure equation may be written as a Poisson equation,

$$
\nabla^{2} \psi=-\Omega
$$

where, $\psi=p$, and,

$$
\Omega=\frac{1}{f_{w} \lambda}\left[\phi \lambda \nabla p \nabla S_{w}+q_{w}-\nabla\left(f_{w} \lambda\right) \cdot \nabla p-\nabla\left(\lambda \nabla p_{c}\right)\right]
$$

note, $\lambda$ is a function of saturation, hence it will vary in both space and time dependant on the saturation distributions on the grid.

As the right hand side involves terms that are dependant on the pressure, these terms must be approximated using information from previous time steps.

\section{Comparison Of Particle Method To Buckley-Leverett Displacement}

In order to test the particle method it is necessary to model two phase flow, which involves the use of relative permeability terms. Relative permeability curves are normally determined experimentally for a particular rock and fluid combination. In this case a theoretical correlation derived by Willhite [1982] is used to simulate the relative permeability data. The equations used are,

$$
\begin{aligned}
& k_{r_{o}}=\left(1-S_{k}\right)^{2.56} \\
& k_{r_{w}}=0.78 * S_{k}^{3.72}
\end{aligned}
$$

where,

$$
S_{k}=\frac{\left(S_{w}-S_{o_{i r}}\right)}{\left(1-S_{w_{i r r}}-S_{o_{i r}}\right)}
$$

Flow field conditions, $\quad S_{w_{i r r}}=0.2$, and, $S_{o_{i r r}}=0.3$.

The fractional flow curve, $f_{w}$, can then be found from,

$$
f_{w}=\frac{1}{1+\frac{k_{r_{0} \mu_{w}}}{k_{r_{w} \mu_{0}}}}
$$

From this data the value of saturation, $S_{w p}$ at which the shock in a Buckley-Leverett displacement will occur may be calculated. 


\subsection{The Buckley-Leverett Equation}

The one dimensional equation describing a two phase system without capillarity or gravity may be written as,

$$
\frac{\partial s}{\partial t}=-q_{T} \frac{\partial f}{\partial x}
$$

This is the Eulerian form of the equation, as it describes the saturation $S$ observed at a point $x$ in space as a function of time, $t$. The Lagrangian form [Fayers \& Sheldon, 1959],

$$
\frac{\partial x}{\partial t}=q_{T} \frac{\partial f}{\partial s}
$$

describes the location, $x$, of a fluid element of saturation $S$ as a function of time; $t$.

Buckley \& Leverett [1942], and, Cardwell [1959] discuss the reason equation (23) yields a triple-valued saturation. As the saturation gradient approaches infinity, prior to the formation of the triple-valued zone, equation (23) becomes invalid. This kind of displacement process is not amenable to solution using differential equations of this type that describe smooth continuous functions.

The use of modified one dimensional random choice methods in order to solve problems in two dimensional reservoir simulation has been investigated by Sethian et al. [1983]. It is shown that characteristic curves representative of the solution of the Buckley-Leverett equation will either form rarefaction waves, Figure 3, or a shock, Figure 4. Each of these conditions is dependant on the shape of the fractional flow, $f_{w}$ curve, and the points on the curve that are sampled to obtain the solution. In the general case the solution is likely to consist of a combination of rarefaction and shock states.

These shocks and rarefactions are also observed when the saturation is being represented by moving particles. Particle re-distribution must be used in order to obtain an accurate representation of the saturation. Farmer [1986] discusses the use of particle control methods when calculating the miscible displacement of passive concentration gradients within a transport fluid.

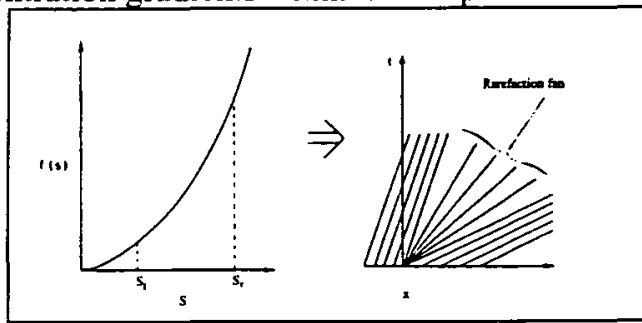

Figure 3. Riemann problem solution for convex $f(s)$ [after Sethian et al. 1983].

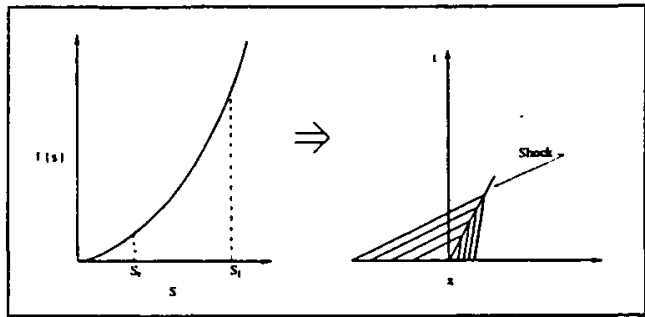

Figure 4. Riemann problem solution for concave $f(s)$ [after Sethian et al. 1983].

\subsection{The Effect Of Capillary Pressure}

Each of the equations (22) and (23) has an equivalent formulation in which the capillary pressure term is included, thus (22) becomes,

$$
\frac{\partial S}{\partial t}+q_{T} \frac{\partial f}{\partial x}+\frac{\partial}{\partial x}\left(f_{w} \frac{\partial P_{c}}{\partial S} \frac{\partial S}{\partial x}\right)=0
$$

and, equation (23) becomes,

$$
\frac{\partial x}{\partial t}=q_{T} \frac{\partial f}{\partial S}+\frac{\partial}{\partial S}\left(\frac{f_{\frac{\partial P}{\partial S}}}{\frac{\partial \vec{\partial}}{\partial S}}\right)
$$

The Eulerian form of the equation, (24), can be solved by replacing the partial differential equation with a finite difference approximation on a grid. As only a single value of $S$ can be represented at each grid node (x), this form of the equation will never become triple-valued. However there is a finite upper limit imposed on the slope that the saturation distribution can attain. The limit is determined by the ratio of the range of values of saturation that are to be represented to the size of the grid spacing. Thus the solution that is calculated will be somewhat smoothed.

It is possible for the Lagrangian form of the Buckley-Leverett equation (23) to become triple-valued, but it has the advantage that it may be used to describe situations with very steep gradients of saturation. Fayers \& Sheldon [1959] demonstrated that inclusion of the capillary term into the Lagrangian formulation, (25), eliminated the triple-valued behaviour of the saturation.

\subsection{Saturation Profiles Obtained Using The Particle Method}

In order to make comparisons with the Buckley-Leverett curves, the two dimensional particle method code was run in a one dimensional mode. Displacement profiles obtained, compared to corresponding Buckley-Leverett profiles, are shown in Figures 5a) and b). These figures were generated using a model that is equivalent to a 10 grid cell one dimensional system. Initially each cell was populated with five particles, evenly distributed, representing a continuous saturation distribution.

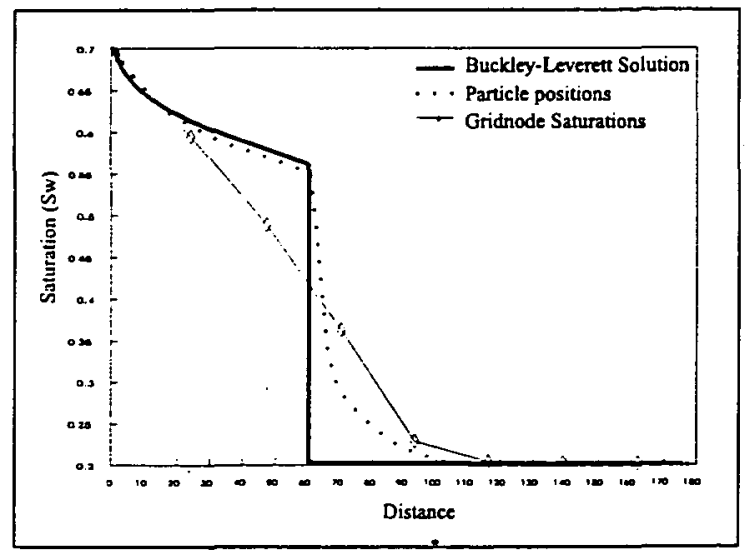

Figure 5. a) Numerical results when considering the saturation to be a smooth continuous function throughout the flow domain. Time step 1 . 


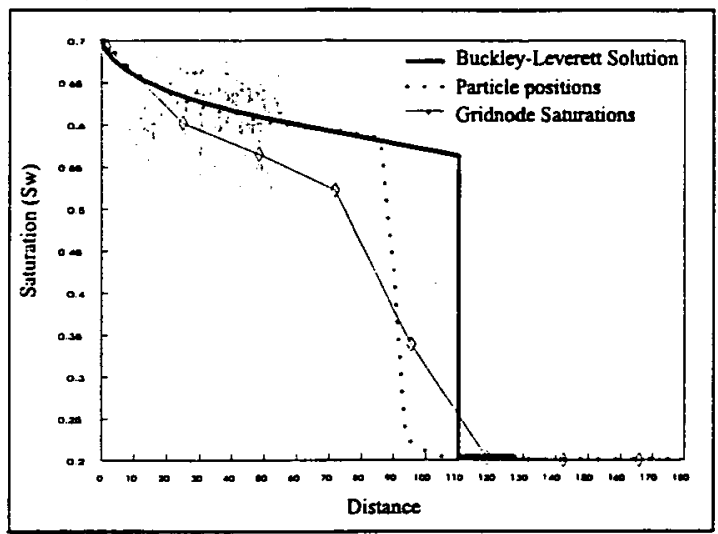

Figure 5. b) Time step 2.

As can be seen from the figures, although the shape of the slowly varying portion of the saturation distribution is correct, the position of the front calculated by the particle method is somewhat retarded in comparison to the true frontal position. This is caused by using a continuous interpolation method on the entire grid. Thus the cells in which there is a discontinuity in saturation, or a very steep saturation gradient will be seen as having a value of saturation that is an average of the particles in the cell. At the shock front there tends to be a compression resulting in the accumulation of many particles covering a large range of saturations. This accumulation of particles in analogous to the convergence of the characteristic curves. The increase in particle concentration introduces averaging errors which must be eliminated in order to accurately predict the velocity of the shock front. This can be achieved by using hybrid interpolation methods or particle insertion and deletion controls.

The hybrid interpolation methods do not necessarily assume that the saturation distribution is a continuous, smooth function, but allow for discontinuities. Farmer [1986b] mentions the use of hybrid interpolation methods that are 'aware' of the prevailing concentration gradients, and adjust accordingly. The use of particle insertion and deletion is most effective when used in combination with a hybridised interpolation scheme.

\subsection{Hybrid Interpolation And Particle Control Methods}

When considering the motion of a discontinuity, the use of an alternative interpolation method in the immediate vicinity of the discontinuity alleviated this problem greatly.

This was achieved in part by the removal of moving points from the flow, when the local saturation gradient became large, as the particles tend to 'bunch up' in these areas. This bunching of particles will cause the cell saturation to becoming averaged, as the default interpolation scheme assumes a smooth distribution of saturation, see Figure 6.

Similarly, moving points must be added to regions where the particle density drops too much. Empty cells (gridblocks containing no particles) occur in areas where the characteristic curves are describing rarefaction waves. The method cannot function correctly. with empty cells, thus these cells must be re-populated. The empty cells will tend to occur in regions where the saturation is varying quite smoothly, thus it is reasonable to interpolate new particles with values of saturation averaged from the surrounding cells.

The sensitivity of the solution to differing methods of particle removal and replacement has also been investigated, and a selection of methods have been evaluated.

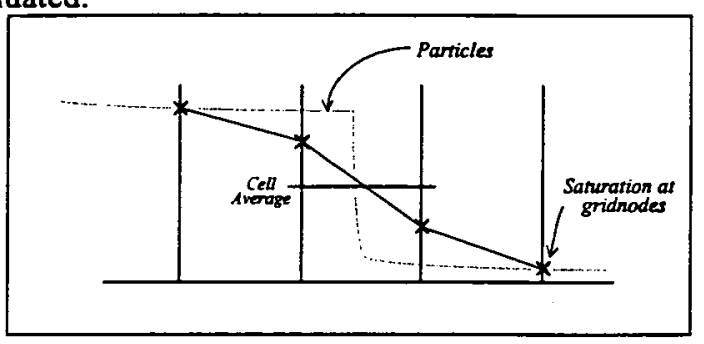

Figure 6. Saturations calculated at gridnodes using smooth (bi-linear) interpolation algorithm throughout the entire domain.

One of the methods mentioned by Farmer [1986b], is the nearest moving point, or NP, interpolation. This method simply assigns to the grid node the value of saturation of the particle that is closest to that point in space.

This method was rejected as, although it deals well with discontinuities, it can cause difficulty in areas of smoothly varying saturation. If, for instance, a : single 'rogue' particle is bypassed by the main flow; or is over-advanced, then it may significantly affect the saturation distribution represented on a particular grid node. The method is not tolerant of even the slightest of errors arising in the distribution of particles. These errors are more likely to arise in the complex situations encountered in reservoir models.

The secondary interpolation method that was chosen to describe the translation of saturation from particles to grid nodes takes into account the occurrence of steep saturation gradients, and deals with the situation appropriately. In most cases it is still possible to use the usual bi-linear area-weighted interpolation method, and this is done for most of the grid. Only in regions where the saturation drops significantly between adjacent grid nodes is the alternative scheme used.

Instead of assuming a smooth saturation transition from one grid node to the next, the alternative scheme respects the existence of a rapid transition. The steep gradient is preserved by allowing the removal of a number of particles that are within approximately $40 \%$ of the cell average, and then interpolating the remaining points onto the grid nodes, by averaging the remaining high values of saturation to the upwind grid node, and the low saturations to the downwind grid node.

A graphical illustration of the technique employed is shown in Figure 7. As can be seen, the saturation distribution is still subject to some averaging, but it is a 
significant improvement over the situation where a smooth saturation distribution is assumed. The averaging effect can be reduced further still by utilising a smaller grid spacing, as this will allow finer resolution of the exact location of the discontinuity.

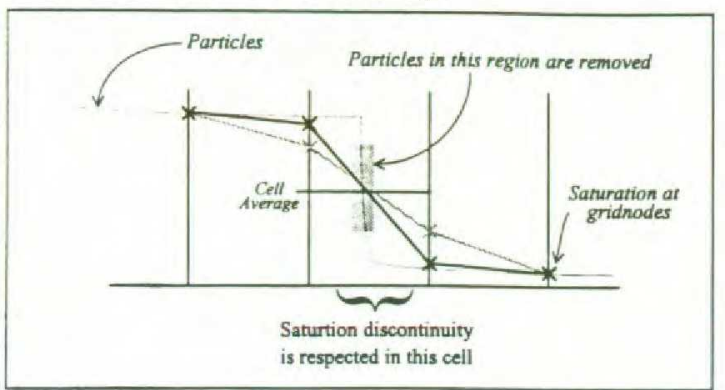

Figure 7. Interpolation of saturation in the region of a discontinuity.

A higher order interpolation method, based on the Gregory-Newton-Taylor (G-N-T) expansion was also investigated by Farmer [1986b]. In view of the results obtained using a combination of the area-weighted interpolation method, and the secondary interpolation scheme described above, the use of the G-N-T method was deemed unnecessary.

\subsection{Results From The Improved Method}

As can be seen from Figures 8a) and b) the results are much improved when using the hybrid interpolation scheme. Both the shape of the saturation distribution and speed of propagation of the shock front are maintained.

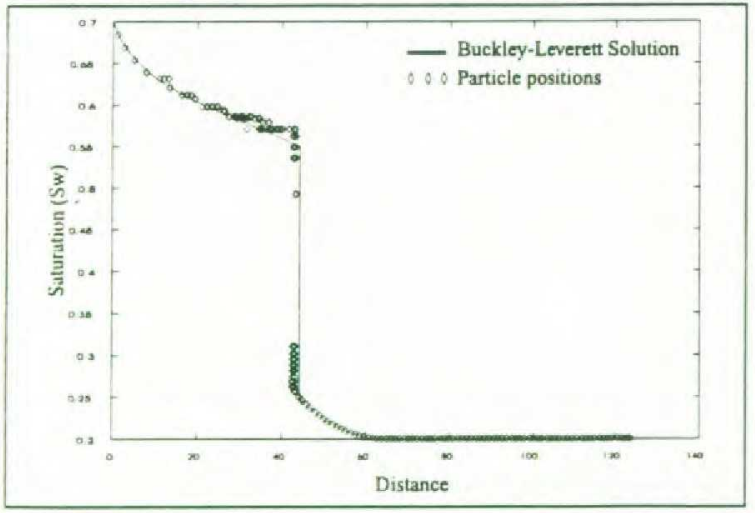

Figure 8. a) Numerical results obtained with hybrid interpolation scheme. Time step 1.

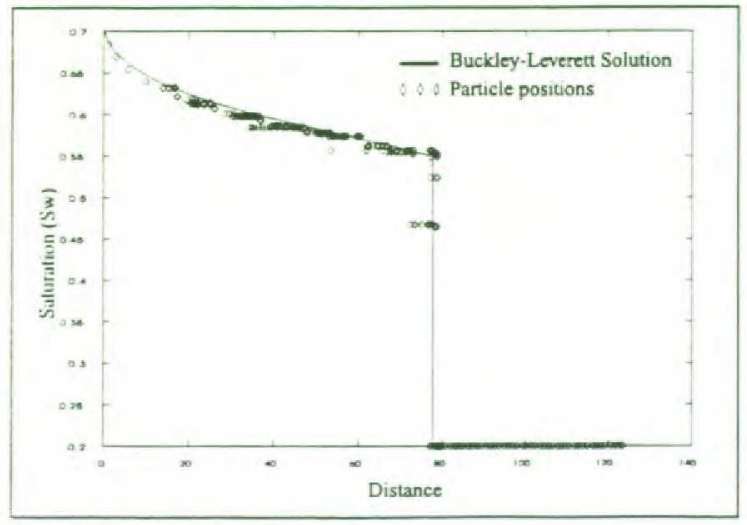

\subsection{Capillary Pressure}

The formation of regions of triple-valued saturations has been avoided by keeping strict control over the formation of the saturation discontinuity front. Particles have been removed in order to prevent averaging effects retarding the motion of the shock front, and, preventing triple-valued saturations occurring in the flow domain.

The model can be used to model flows more accurately by including the capillary pressure terms into the pressure equation. In order to investigate the effects of the capillary pressure on the evolution of the saturation distribution, a non-zero capillary pressure curve is entered into pressure solver code. The same data as used by Fayers \& Sheldon [1959] was used in the numerical experiments done here.

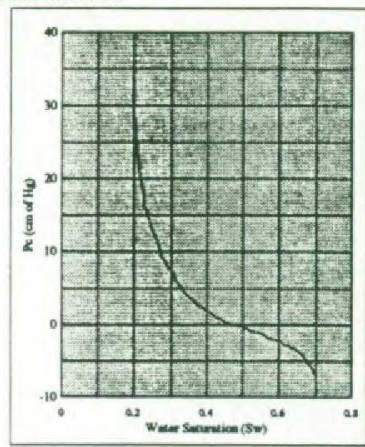

Figure 9. Capillary pressure curve used in the particle modelling code.

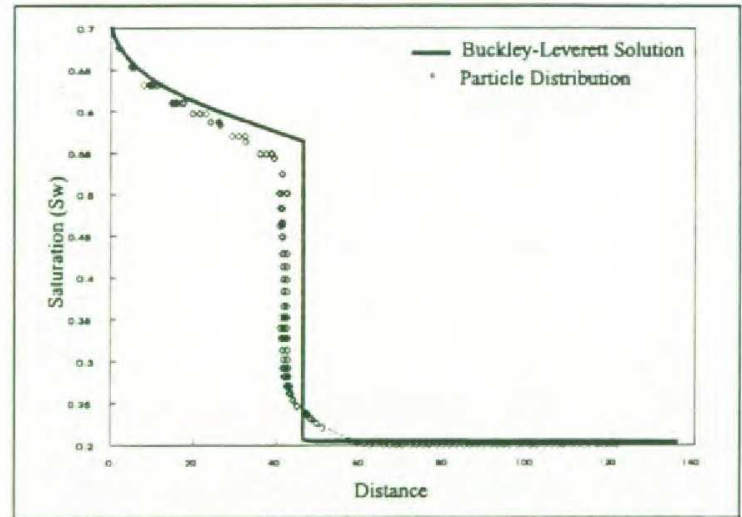

Figure 10.Effect of capillary pressure on the formation of saturation shock fronts.

With the introduction of the capillary pressure term, the displacement in no longer a simple Buckley-Leverett displacement. The solution does not degenerate to a triple valued saturation, as described by Fayers \& Sheldon [1959].

The speed of advancement of the 'discontinuity' is also slightly reduced. This is a numerical artefact, requiring further investigation, caused by the averaging of the capillary pressure gradient terms on a grid with finite spacing. In an error-free method an averaging of the front in the near vicinity of the shock would be observed.

Figure 8. b) Time step 2. 


\section{Two Dimensional Comparison, An Experimental Validation}

The test case is based on the experiments of Beadie \& Dawe [1981]. The experimental displacement is shown in Figure 11.a) and may be compared to the results obtained by the particle method shown in Figure 11.b). The particle method model utilised a $15 \times 15$ cell grid, initially populated with approximately 36 particles in each cell, a total of 8,100 particles. This is still quite a low number of particles for a method of this type; aeronautical engineering codes often utilise several hundreds of thousands of particles to characterize turbulent flow, Graham [1994].

As can be seen from Figures $\mathbf{1 1}$ a) and b) as the fluid is displaced through the model, some of the fluid is 'retained' in the low permeability zones. This phenomenon is observed in the corresponding areas of the experimental displacement.

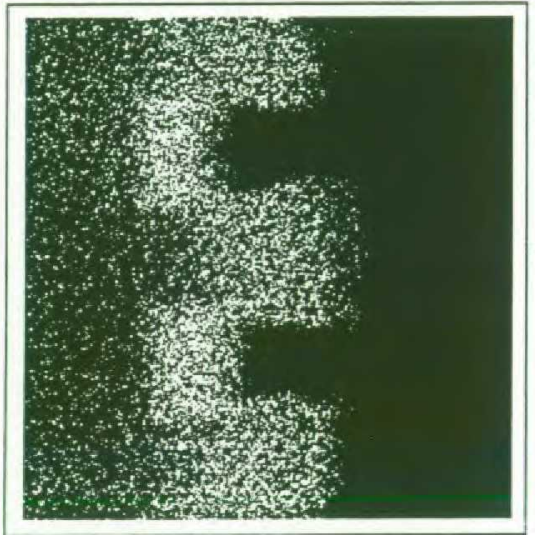

Figure 11.a) Experimental Result [after Beadie \& Dawe, 1981].

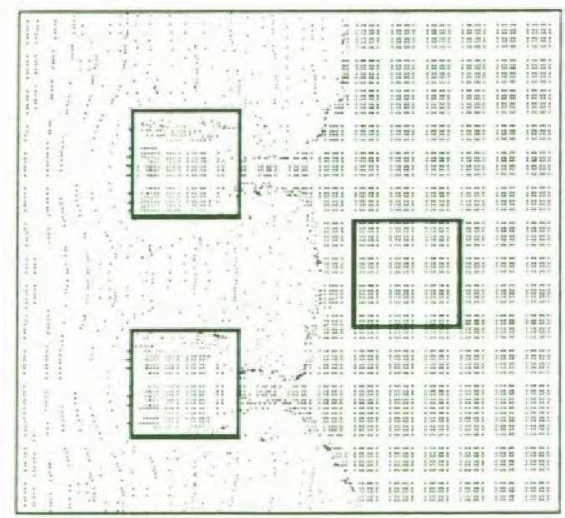

Figure 11. b) Particle Method Result.

Also demonstrated in Figures $\mathbf{1 1}$ a) and b) is the ability of the particle method to resolve the characteristics of the fluid flow below the level of an equivalent coarse finite difference grid. This improvement is achieved because the particles are free to move in any direction; they are not constrained to move along the grid lines.

\section{Extension To Three Dimensions}

Many of the concepts used in the two dimensional particle method are directly extensible to the three dimensions. Only some variations in the particle control methods are required to obtain a fully working model.

The solver used to calculate the solution of the pressure equation in three dimensions was supplied by Daltaban [1994]. The code used is a proven, stable, code that has been used and tested extensively in a finite difference reservoir simulator.

\subsection{Interpolation And Particle Control}

In order to carry out smooth interpolation of saturation from particle to surrounding grid nodes, a volume weighting scheme is utilised, see Figure 12. The scheme is similar in principle to the two dimensional bilinear area weighting. The contribution of each particle to the value of saturation at a particular node is calculated as follows. The cell in which the particle rests is divided into eight segments, each of volume $V_{i}, i=1, \ldots, 8$. Each cell is of volume $V_{\text {cell, }}$, thus,

$$
V_{\text {cell }}=\sum_{i=1}^{8} V_{i}
$$

Each particle, of saturation $S_{\text {part }}$, contributes to the value of saturation at each of the grid nodes in the following proportions,

$$
S_{\text {node }_{i}}=S_{\text {part }} \frac{V_{i}}{V_{\text {cell }}}
$$

This contribution of each particle to the value of saturation at each grid node is also weighted in proportion to the total number of particles in the immediate area of the grid node. The same interpolation method is also used in order to transfer velocity from grid nodes back to particles.

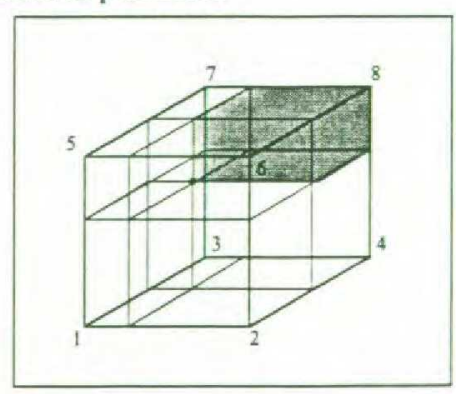

Figure 12.Opposite volume weighting interpolation scheme.

The particle control methods remain substantially similar to those used in two dimensions. The only differencing being that now the direction of $\nabla p$ must be considered in three dimensions rather than just two. Also the concept of a discontinuity in saturation must be extended to three dimensions, which is somewhat more difficult to visualize, but computationally quite similar to the two dimensional case. 


\section{Combination With The Boundary Integral Method}

When calculating the solution to the pressure equation, or velocity vector, within a flow domain, it is important to consider the effects of rock inhomogeneity and fluid inhomogeneity simultaneously. Both changes in the properties of the porous media and the fluid can have drastic effects on the flow of fluid in the model. Neglecting one or the other can lead to highly erroneous results being output from the simulation.

Thus to combine the boundary integral and particle methods, the influence of the particles representing saturation on the interfaces representing permeability heterogeneity must be calculated when the interface equations are solved. A technique is proposed to attempt this combination of methods in order to produce a simulation technique that makes best use of the strengths of each of the methods.

Roberts \& Christiansen [1972] mention methods of dealing with discontinuities in fluid properties by representing interfaces by a polygon consisting of marker points joined by straight-line segments. The marker points are convected with the fluid, and the shape of the polygon defining the surface allows the calculation of the area of each fluid lying within a given mesh cell. Using this information it is possible to define an average equation of state with a cell.

The preferred method of computing the motion of a discontinuity is shown in Figure 13a) to c). The inhomogeneity within the flow field, and the lines joining the particles defining the vertices of the polygon describing the interface between two fluid phases are represented using vortex panels. There are a number of problems associated with this method due to the constant re-arrangements of the vortex panels defining the interface. The sequence of Figures 13a) to c) describes the interaction of a fluid front with a homogeneity in the permeability distribution.

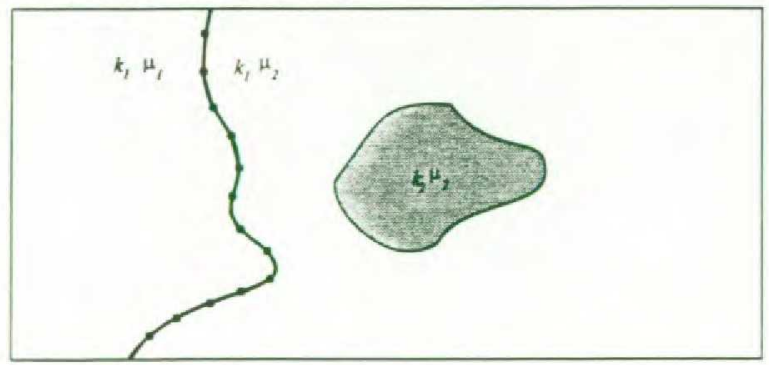

Figure 13.a) Time step 1.

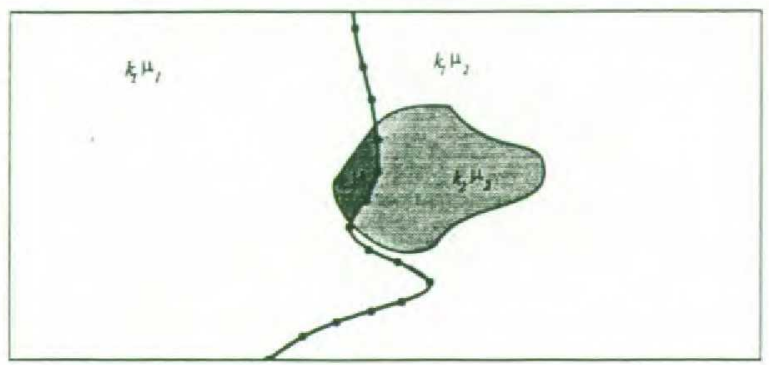

Figure 13. b) Time step 2.

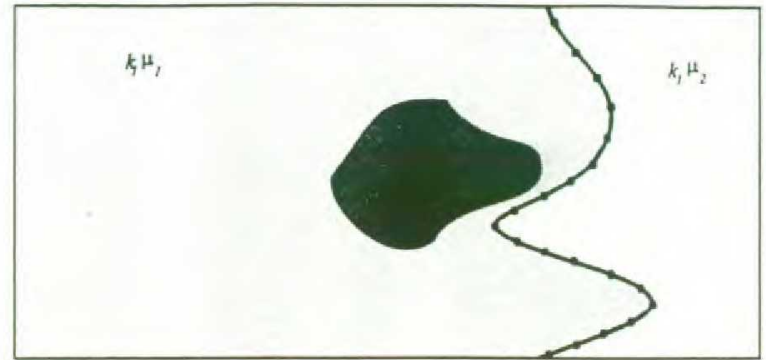

Figure 13. c) Time step 3.

As the fluid front encounters the inhomogeneity the combination of fluid interface (describing a discontinuity in fluid properties), and, the surface of the body (describing a discontinuity in permeability) form new zones depending on the combination of fluid and porous medium at a particular point in space and time. Figure 13b) is composed of four distinct zones with different sets of permeability and fluid properties.

It is necessary to treat the interfaces with the boundary conditions appropriate to the discontinuities in properties being described. Although this re-zoning of the flow system appears to be quite trivial, it is difficult to achieve computationally whilst still maintaining some degree of efficiency in the method. This method must be combined with some form of domain decomposition in order to be viable.

An alternative solution is to make use of the coarse grid overlaid onto the particles. The saturation is characterized by the particles, and each cell can be given a representative value for its average saturation. Using a Green's function to represent the distribution of saturation at the cell centres it may be possible to calculate the effect of the saturation distribution on the panels defining the permeability discontinuities in the boundary integral method calculation. Once the boundary integral method calculation has been completed, the particles move to their new positions.

A direct calculation of the influence of every panel on every particle is not feasible, as this would involve $\mathrm{O}\left(N_{\text {particles }} N_{\text {panes }}\right)$ operations to complete the convection of all the particles. It is thus necessary to use an approximate method of some kind to calculate the motion of the particles.

The velocity may be transformed from the grid nodes and then from the grid nodes to the particles, as before. This method would result in a total of $\mathrm{O}\left(N_{\text {panels }} N_{\text {nodes }}+4 N_{\text {particles }}\right)$ calculations, which is less than the direct calculation. However the transformation of velocities from the panels to the grid nodes may be computationally intensive if the total number of nodes $N_{\text {nodes }}$ is large. In these cases it is necessary to adopt a further approximation technique by lumping the effects of some panels in a domain decomposition method. This may be achieved for instance, by only directly calculating the effects of the panels in the near vicinity of a grid node (say within one cells distance). The contributions of these panels to distant grid nodes may 
then be deduced from the value of the velocity calculated for the node to which the panels are closest.

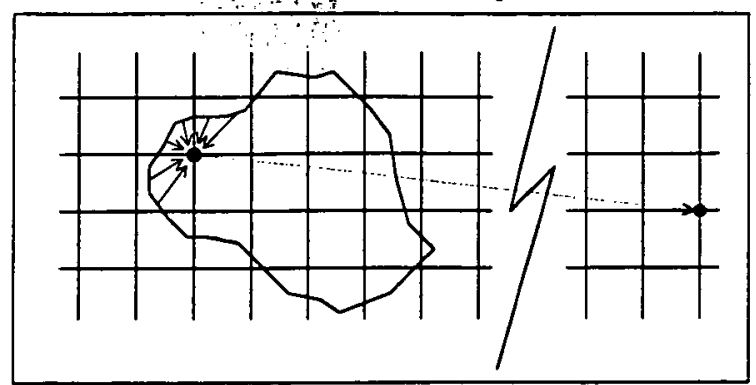

Figure 14. Calculation of velocities at grid nodes from boundary integral panels.

\section{Conclusions}

This combined fixed grid and moving particle technique has been used to numerically reproduce analytic solutions of the one-dimensional Buckley-Leverett equation. Comparisons have also been made with experimental two-dimensional displacements. The extension of the method to modelling three dimensional displacements is also investigated.

1. The advantage of this approach over conventional fixed mesh methods is that the saturation distribution can be more accurately characterised without significant additional cost in computational effort.

2. The boundary integral aspect of this method is particularly useful for a class of problems involving immiscible displacements in highly heterogeneous media.

3. Particles are used to characterise saturation, in three dimensions, rather than concentration, as has been done previously.

\section{References}

Aziz, K. \& Settari, A. : "Petroleum Reservoir Simulation", Elsevier Science Publishers Ltd., 1979.

Basuki,J: Unsteady Flows Over Aerofoils and Cascades, $\mathrm{PhD}$ Thesis, Department of Aeronautical Engineering, Imperial College, University of London, 1983.

Beadie \& Dawe, R.A. : Project Work, carried out at Imperial College, University of London, 1981.

Birdsall, C.K. \& Fuss, D. : Clouds-in-Clouds, Clouds-in-Cells, Physics for Many-Body Plasma Simulation, Journal of Computational Physics 3, 1969.

Buckley, S.E., \& Leverett, M.C. : Mechanism of Fluid Displacement in Sands, T.P. 1337, Petroleum Technology, May, 1941.

Cardwell, W.T. : The Meaning of the Triple Value in Noncapillary Buckley-Leverett Theory, Petroleum Transactions, AIME, volume 216, 1959.

Christiansen, J.P. : Numerical Simulation of Hydrodynamics by the Method of Point Vortices, Joumal of Computational Physics 13, Academic Press, 1973.
Cooley, J.W. \& Tukey, J.W. : An Algorithm for the Machine Calculation of Complex Fourier Series, Journal of Mathematics of Computation, Volume 19 (April), p. 297, 1965.

Daltaban, T.S. : Personal Communication, 1994.

Farmer, C.L. : A Moving Point Method for the Numerical Calculation of Miscible Displacement, Proceedings of IFE-SSI Seminar on Reservoir Description and Simulation, w. Emphasis on EOR, 1986.

Farmer, C.L. \& Norman, R.A. : The Implementation of Moving Point Methods for Convection-Diffusion Equations, in "Numerical Methods for Fluid Dynamics II", Editors: Morton, K.W. \& Baines, M.J. , Clarendon Press, Oxford, 1986a..

Farmer, C.L. : Unpublished report. 1986b.

Fayers, F.J. \& Sheldon, J.W. : The Effect of Capillary Pressure and Gravity on Two-Phase Fluid Flow in a Porous Medium, Petroleum Transactions, AIME, Volume 216, 1959.

Felix, J.P.M. : Computer Modelling of Vortex Meter Flowfields Using the Discrete Vortex Method, PhD Thesis, Department of Aeronautical Engineering, Imperial College, University of London, 1987.

Graham, J.M.R. : Computation of Viscous Separated Flow Using a Particle Method, in "Numerical Methods for Fluid Dynamics III", IMA, 1988.

Graham, J.M.R. : Personal Communication, 1994.

Homolka, D.T. : The Application of Boundary Integral and Particle Methods in Modelling Fluid Displacement in Petroleum Reservoirs, $\mathrm{PhD}$ Thesis, Department of Mineral Resources Engineering, Imperial College, University of London, 1995 (Unpublished to date).

Pietlicki, R. \& Archer, J.S. : Novel Scheme for Control of Numerical Diffusion in Reservoir Simulation, Chem. Eng. Res. Des., Vol. 65, January, 1987.

Raviart P.A. : Particle Numerical Models In Fluid Dynamics, in "Numerical Methods for Fluid Dynamics II", Editors: Morton, K.W. \& Baines, M.J. , Clarendon Press, Oxford, 1986.

Roberts, K.V. \& Christiansen, J.P. : Topics in Computational Fluid Mechanics, Computer Physics Communications 3, Suppl. (1972), North-Holland Publishing Company, 1972.

Sethian, J.A., Chorin, A.J., \& Concus, P. : Numerical Solution of the Buckley-Leverett Equations, SPE 12254, Reservoir Simulation Symposium, San Francisco, 1983.

Willhite, G.P. : "Waterflooding", SPE Textbook Series, Volume 3, 1986.

Wong, D.W. : A Novel Approach to Model Immiscible Displacements Through Heterogeneous Porous Media, $\mathrm{PhD}$ Thesis, Department of Mineral Resources Engineering, Imperial College, Univ. of London, 1991. 